\title{
O USO INADEQUADO DO MISOPROSTOL COMO ABORTIVO: UMA REVISÃO INTEGRATIVA
}

Mariana Fernandes Esteves, Rafael da Silva Sá, Natalia Maria Rodrigues, Rafaela Borges Batalha, Melina Ariane Calamita Quiroga

Universidade do Oeste Paulista - UNOESTE, Faculdade de Medicina, Presidente Prudente, SP. E-mail: marianafer.est@gmail.com

\section{RESUMO}

Devido à ocorrência frequente do aborto ilegal no Brasil e em países onde a prática é criminalizada, o medicamento misoprostol vem sendo um meio de fácil acesso por mulheres que buscam interromper uma gravidez. Todavia, muitas vezes o seu uso é realizado de forma incorreta e o aborto acaba não sendo efetivo. Deste modo, o objetivo do estudo foi reunir os dados disponíveis na literatura para analisar o impacto do uso inadequado do misoprostol na saúde materna e fetal. A revisão integrativa foi adotada para realização deste trabalho. Foram incluídos artigos que abordam a temática do aborto inadequado com o uso de misoprostol, dentro de todas as áreas de interesse da saúde e excluídos aqueles cuja temática expõe outros fins terapêuticos do fármaco. Assim como visto na literatura, o uso incorreto e inconsciente do misoprostol pode causar prejuízos graves à saúde materna e fetal, embora seja comprovada cientificamente a sua segurança em meios hospitalares com respaldo de indicação médica e de profissionais capacitados.

Palavras-chave: aborto, misoprostol, riscos teratogênicos, malformações congênitas, síndrome de Mobius.

\section{IMPROPER USE OF MISOPROSTOL AS ABORTIVE: AN INTEGRATIVE REVIEW}

\begin{abstract}
Due to the frequent occurrence of illegal abortion in Brazil and in countries where the practice is criminalized, the drug misoprostol has been a means of easy access for women who seek to terminate a pregnancy. However, its use is often performed incorrectly and abortion ends up not being effective. Thus, the aim of the study was to gather the data available in the literature to analyze the impact of the inflationary use of misoprostol on maternal and fetal health. The integrative review was adopted to carry out this work. Articles that address the theme of inappropriate abortion with the use of misoprostol were included, within all areas of health interest, and those whose theme exposes other therapeutic purposes of the drug were excluded. As seen in the literature, the incorrect and unconscious use of misoprostol can cause serious damage to maternal and fetal health, although it is scientifically proven to be safe in hospitals with the support of medical indication and trained professionals.
\end{abstract}

Keywords: abortion, misoprostol, teratogenic risks, congenital malformations, Mobius Syndrome.

\section{INTRODUÇÃO}

O fármaco misoprostol é uma substância análoga da prostaglandina E1 (PGE1), cuja principal indicação, inicialmente, foi prevenir úlceras gastro-duodenais. Posteriormente, foi empregado como estimulante uterino, induzindo contrações e alargamento do colo uterino. Logo, percebeu-se seu poder abortivo, sendo introduzido em práticas hospitalares e também ilegais. Nos dias atuais, tem sido indicado na indução de aborto legal, no esvaziamento uterino por morte embrionária ou fetal, no 
amolecimento cervical antes de aborto cirúrgico, na estimulação de trabalho de parto, no controle da hemorragia pós-parto, e para infundir modificações na cérvice uterina, facilitando a realização de procedimentos, como histeroscopia e colocação de dispositivo intrauterino ${ }^{1}$. No entanto, as práticas ilegais contribuíram para consequências severas na saúde tanto das gestantes quanto de seus fetos. O medicamento foi introduzido no Brasil no ano de 1986 e sua comercialização não possuía nenhuma restrição. Após alguns anos, em 1991, quando descobriram que possuía propriedades abortivas, tornou-se um método de fácil acesso, e isso acabou limitando sua comercialização apenas com prescrição médica ${ }^{2}$.

Em 1998, a Agência Nacional de Vigilância Sanitária (ANVISA) delimitou o acesso ao medicamento misoprostol apenas aos estabelecimentos hospitalares, impedindo sua disponibilidade nas farmácias. Atualmente, no Brasil, a comercialização do misoprostol está proibida em farmácias de todo o país, por determinação da Portaria 344/98 do Ministério da Saúde, que regulamenta a distribuição de medicamentos sujeitos a controle especial. Com os nomes comerciais de Cytotec $^{\circledR}$ e Prostokos $^{\circledR}$, segue sendo autorizado apenas para uso hospitalar, utilizado por profissionais de saúde na indução do parto ou para interrupção da gravidez prevista em lei, de acordo com a Norma Técnica de Atenção Humanizada ao Abortamento e a Norma Técnica de Prevenção e Tratamento dos Agravos Resultantes da Violência Sexual do Ministério da Saúde ${ }^{3}$.

No Brasil, embora os dados sobre o aborto sejam incompletos, o Sistema de Informações sobre Mortalidade (SIM) do país registrou entre 2006 e 2015, 770 óbitos com causa básica aborto. Em países que possuem restrições legais ao aborto, como no Brasil, as mulheres recorrem ao aborto inadequado tornando-o uma das principais causas de morbimortalidade materna, principalmente quando relacionados com métodos e pessoas não qualificados ${ }^{5}$.

Já em países como Rússia, México, Polônia, Islândia, Espanha, Suécia, Japão, Noruega, Estados Unidos, Reino Unido, Canadá, Austrália, Índia, França, Alemanha e Irlanda a prática é legalizada, de forma que consideram a escolha reprodutiva uma ferramenta básica para garantir os direitos humanos da mulher. Porém, no Brasil, o aborto é tido como um crime contra a vida, conforme previsto no Código Penal Brasileiro, em que há três exceções onde é permitido: risco de vida à gestante, tido como aborto necessário; gravidez ocasionada por estupro, correspondendo ao aborto humanitário; e se o feto for anencéfalo. Em casos de anomalia fetal incompatível com a vida, também têm sido atendidos nos serviços de saúde, mediante autorização judicial ${ }^{6}$.

A Organização Mundial de Saúde (OMS) define aborto inseguro como um procedimento para o término da gestação, realizado por pessoas sem a habilidade necessária ou em um ambiente sem padronização para a realização de procedimentos médicos, ou a conjunção dos dois fatores ${ }^{3}$. Segundo a Federação Internacional de Ginecologia e Obstetrícia (FIGO), a cada ano, 25 milhões de mulheres e meninas em todo o mundo sofrem abortos inseguros, contribuindo para uma estimativa de $13 \%$ da mortalidade materna e resultando em lesões a longo prazo e infertilidade para milhões a mais. Esses números representam um problema evitável, mas muitas vezes negligenciado, de saúde pública e de direitos das mulheres ${ }^{2}$.

Diante da eficácia do misoprostol, a Organização Mundial de Saúde (OMS) incluiu-o na lista de medicamentos essenciais, o que também foi acatado pelo Brasil ao introduzi-lo na Relação Nacional de Medicamentos Essenciais do Ministério da Saúde (RENAME/ MS - 2010). Apesar da restrição ao medicamento, sabe-se que jovens e mulheres realizam aborto com misoprostol por meio de acesso irregular ao mesmo, comprando-o no mercado paralelo (ilegal), submetendo-se a utilizar medicamentos falsificados (substâncias com efeito e origem desconhecidas), com pouca ou nenhuma informação sobre as indicações de uso, tampouco sobre as formas de administração, colocando em risco sua saúde reprodutiva ${ }^{3}$.

Devido à ocorrência frequente de aborto ilegal no Brasil, o misoprostol vem sendo um meio de fácil acesso e fácil administração pelas usuárias, porém, muitas vezes o seu uso é realizado de forma incorreta e o aborto acaba não sendo efetivo, resultando na morte do feto ou em consequências teratogênicas, além de impactos na saúde da mulher. Portanto, esse estudo visa analisar as consequências geradas através do uso inadequado do misoprostol, mostrando também a importância do amparo médico para evitar tais desfechos, visto que o ato ocorrerá independente de sua legalidade. 
O presente estudo tem como objetivo analisar, por meio de uma revisão integrativa, o uso inadequado do misoprostol, seus possíveis riscos teratogênicos e maternos; verificar o modo de administração do misoprostol e correlacionar com o aborto; analisar as consequências para o feto como doenças e síndromes em decorrência da administração de misoprostol como abortivo sem acompanhamento médico; e considerar que o uso inadequado do misoprostol pode acarretar consequências à saúde da mulher, e, consequentemente, afetar o sistema de saúde pública.

\section{MÉTODOS}

Trata-se de um estudo secundário de pesquisa sobre os riscos do uso incorreto do misoprostol como abortivo. A revisão integrativa foi adotada para realização deste trabalho, visto que possibilita sumarizar as pesquisas já concluídas e obter conclusões a partir do tema de interesse, pois associa em um único estudo os riscos teratogênicos e maternos mais comuns em consequência do uso inadequado desse medicamento.

Para a realização deste estudo, foram seguidas as seis etapas de uma Revisão Integrativa: 1) seleção da pergunta norteadora da pesquisa; 2) busca na literatura a partir da pergunta; 3) definição dos critérios para a coleta de dados; 4) análise crítica dos estudos selecionados, considerando todas as características em comum com a pergunta; 5) discussão dos resultados encontrados; 6) união dos dados e elaboração da revisão integrativa.

A pergunta que norteou esta pesquisa foi: Quais os possíveis riscos teratogênicos e maternos ao uso inadequado de misoprostol como abortivo? Devido ao grande número de consequências que um aborto sem assistência médica e mal realizado pode causar, muitas mulheres se submetem e se arriscam na realização deste procedimento, que pode gerar não só consequências à elas, mas também aos fetos.

Deste modo, para a análise dos dados encontrados, foram adotados os seguintes critérios para seleção dos artigos: estudos de relevância em que responderam à pergunta norteadora a qual abrange os prováveis riscos maternos e fetais em consequência do uso inadequado de misoprostol; artigos publicados em periódicos nacionais e internacionais de língua inglesa, espanhola e francesa; artigos que abordem a temática do aborto ilegal, dentro de todas as áreas de interesse da saúde; periódicos indexados nas bases de dados utilizadas. Foram excluídos artigos cuja temática expõe outros fins terapêuticos desse medicamento; artigos de relatos de casos, estudos com metodologias indefinidas, cartas e comentários.

Segue abaixo um fluxograma organizado para nortear a seleção de artigos encontrados. 


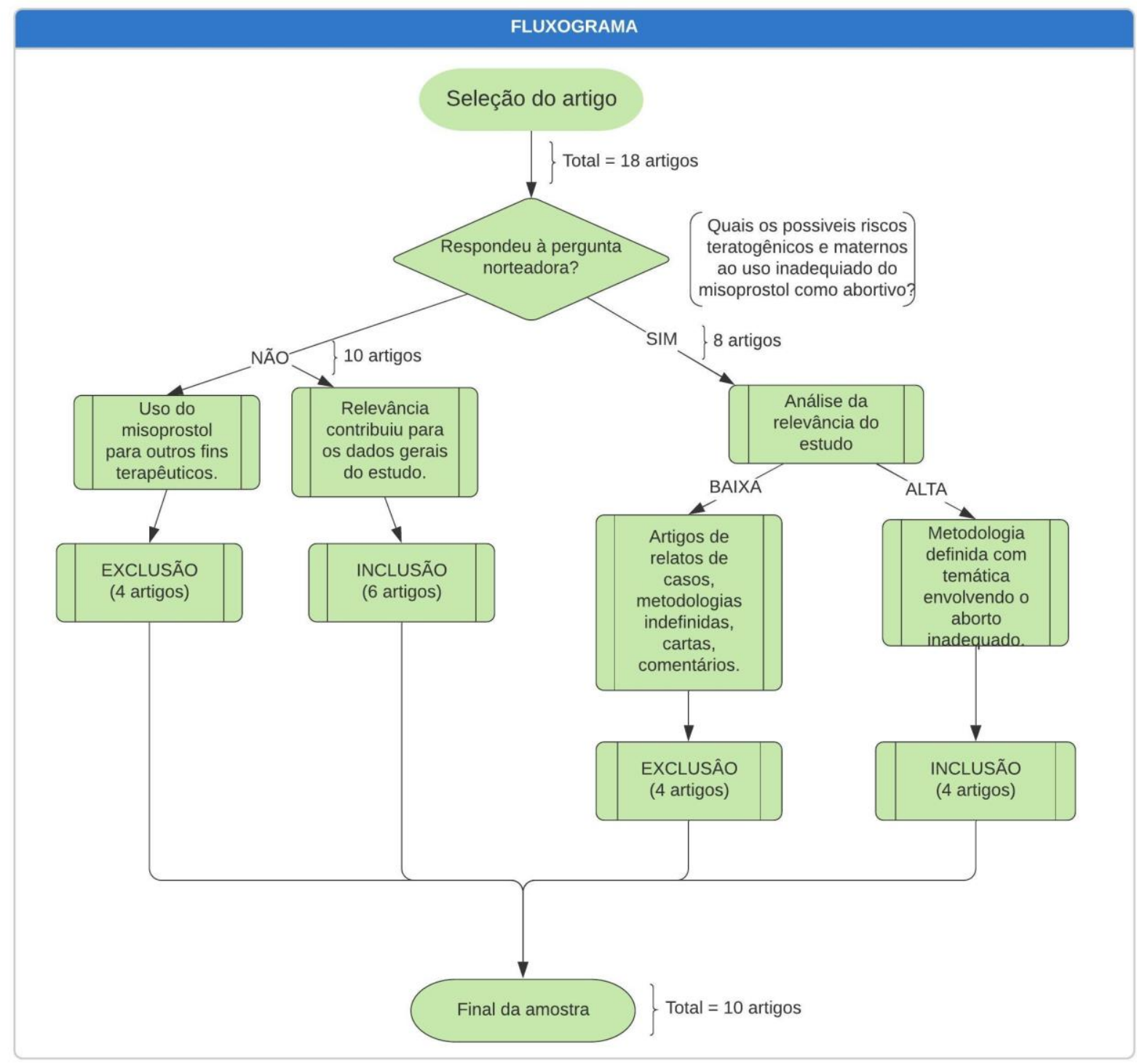

Figura 1. Fluxograma para a seleção de artigos.

Fonte: Próprio Autor (2020).

Para a coleta dos artigos foram empregadas as bases de dados PubMed, Scielo, Biblioteca Virtual em Saúde (BVS), Scopus, LILACS e EMBASE. E para o levantamento dos artigos utilizaram-se as palavras-chave "misoprostol", "aborto", "riscos teratogênicos", "abortivos". Não houve restrição de datas de publicação.

Após a leitura na íntegra dos artigos, foi utilizado como ferramenta de coleta dos dados um instrumento validado por Ursi e al., o qual compõe itens como: objetivo ou questão de investigação; amostra; tratamento dos dados; intervenções realizadas; resultados; implicações; níveis de evidências; avaliação do rigor metodológico. Dessa forma, ao serem catalogados, os estudos foram classificados em seis diferentes níveis de evidência, sendo: Nível 1: evidências resultantes da meta-análise de múltiplos estudos clínicos controlados e randomizados; Nível 2: evidências obtidas em estudos individuais com delineamento experimental; Nível 3: evidências de estudos quase-experimentais; Nível 4: evidências de estudos descritivos (não-experimentais) ou com abordagem qualitativa; Nível 5: evidências provenientes de relatos de casos ou de experiência; Nível 6: evidências baseadas em opiniões de especialistas.

Seguiu-se com a elaboração de uma tabela contendo título do artigo, autores, tipo e origem do estudo, intervenção estudada, resultados e recomendações/conclusões. Por fim, foi possível comparar criticamente os resultados 
entre todos os estudos selecionados e discutir os vieses encontrados.

\section{RESULTADOS}

Nesta revisão integrativa, encontraram-se dezoito artigos que atenderam à temática proposta relacionada ao uso inadequado do misoprostol, porém oito foram excluídos por falta de relevância do estudo (relatos de casos, metodologias indefinidas, cartas e comentários) ou porque se relacionaram ao uso de misoprostol para outros fins terapêuticos não associados ao aborto. No final da amostra, analisaram-se dez artigos que atenderam aos critérios já descritos e previamente estabelecidos, definiram-se suas relevâncias e metodologias e coletaram-se os dados para a elaboração da revisão. A seguir, será apresentado um prisma geral dos estudos avaliados.

Em relação ao delineamento de pesquisa dos artigos inclusos na revisão notabilizaram-se na amostra: três estudos de revisão sistemática; três estudos observacionais, sendo dois de caráter prospectivo e um de caráter retrospectivo; um estudo transversal; um estudo experimental em animais; um estudo de caráter qualitativo e um estudo com delineamento de pesquisa não-experimental. Em tal configuração, quanto ao nível das evidências obtidas, encontraram-se três artigos com o nível 1 ; três artigos com o nível 2; um artigo com o nível 3; e três artigos com o nível 4.

Quanto ao tipo de revista em que foram publicados os artigos da revisão, majoritariamente revistas médicas internacionais cujos trabalhos publicados se inserem nos campos de ginecologia, obstetrícia, saúde reprodutiva e saúde da mulher. Apenas um dos artigos foi publicado em um periódico acadêmico de acesso aberto.

A seguir, na tabela 1, apresenta-se a síntese dos artigos da amostra presente na revisão integrativa.

Tabela 1. Apresentação da síntese de artigos incluídos na revisão integrativa.

(continua)

\begin{tabular}{|c|c|c|c|c|c|}
\hline $\begin{array}{l}\text { Nome do } \\
\text { artigo/Autores }\end{array}$ & $\begin{array}{l}\text { Tipo/Origem } \\
\text { estudo }\end{array}$ & do & $\begin{array}{l}\text { Intervenção } \\
\text { estudada }\end{array}$ & Resultados & $\begin{array}{l}\text { Recomendações/ } \\
\text { Conclusões }\end{array}$ \\
\hline
\end{tabular}

Preventing unsafe Estudo ecológico abortion:

Achievements Federação and challenges of a global FIGO initiative Internacional de Ginecologia e Obstetrícia (FIGO)
Prevenção do aborto envolvendo 46 sociedades membros de diferentes países, constando uma análise situacional e preparo de um plano de ação com base nos resultados.

Prevalence,
attitudes and
knowledge of
misoprostol for
self-induction of
abortion in
women
presenting for
abortion at

$\begin{array}{ll}\text { Estudo transversal } & \begin{array}{l}\text { Aumento da } \\ \text { prevalência de }\end{array} \\ \text { University of lowa } & \text { mulheres que } \\ \text { Hospitals and } & \text { procuram ou } \\ \text { Clinics, lowa City, } & \text { empregam } \\ \text { IA, Estados Unidos } & \text { misoprostol para } \\ & \begin{array}{l}\text { aborto auto- } \\ \text { induzido. }\end{array}\end{array}$

Considerando que os países envolvidos possuíam muitas culturas, religiões e constituições políticas diferentes, foram propostas estratégias alternativas correspondentes a quatro níveis de prevenção

Mulheres que
investigaram o
misoprostol
provavelmente
usariam essa
opção (38,2\% vs.
$20,7 \%, p=.27)$, e
consideraram que
aumentaria o

Melhorar o acesso a serviços de aborto seguro NÃO aumenta a taxa de aborto, mas é um instrumento importante para reduzi-la, ao mesmo tempo que reduz a morbidade e mortalidade associada ao aborto clandestino.

A maioria das
mulheres que
procuraram por um
aborto faziam a
busca online antes
de irem à consulta e
um terço delas
investigou o
misoprostol para

A maioria das mulheres que procuraram por um aborto faziam a busca online antes de irem à consulta e investigou o misoprostol para 


\author{
reproductive \\ health clinics \\ Kerestes, $\mathrm{C}$ \\ Sheets, K \\ Stockdale,C \\ HardyFairbanks,A
}

$\begin{array}{ll}\text { acesso ao aborto } & \text { uso doméstico, } \\ (53,7 \% \text { vs. } 36,4 \%, & \text { sugerindo que a } \\ \mathrm{p}=0,28) .0 & \text { disponibilidade e } \\ \text { misoprostol havia } & \text { possivelmente o } \\ \text { sido comprado } & \text { desejo de abortos } \\ \text { por } 1,6 \% \text { das } & \text { auto-induzidos com } \\ \text { entrevistadas. } & \text { misoprostol estão } \\ & \text { aumentando. }\end{array}$

acesso ao aborto uso doméstico,

$(53,7 \%$ vs. $36,4 \%$, sugerindo que a

$\mathrm{p}=0,28)$. 0

misoprostol havia

sido comprado

desejo de abortos

misoprostol estão

aumentando.

Tabela 1. Apresentação da síntese de artigos incluídos na revisão integrativa.

\begin{tabular}{ll}
\hline $\begin{array}{l}\text { Nome do } \\
\text { artigo/Autores }\end{array}$ & $\begin{array}{l}\text { Tipo/Origem do } \\
\text { estudo }\end{array}$ \\
\hline $\begin{array}{l}\text { Complications } \\
\text { with use of } \\
\text { misoprostol for } \\
\text { abortion in }\end{array}$ & Madudagascar \\
Madagascar: \\
between ease of \\
access and lack of \\
information
\end{tabular}

$\begin{array}{ll}\begin{array}{l}\text { Commercial } \\ \text { availability of } \\ \text { misoprostol and } \\ \text { induced abortion } \\ \text { in Brazil }\end{array} & \begin{array}{l}\text { Revisão } \\ \text { sistemática }\end{array} \\ & \begin{array}{l}\text { Fundação Ford, } \\ \text { Escola Nacional } \\ \text { de Saúde Pública, }\end{array} \\ \text { Costa, S.H } & \begin{array}{l}\text { Rio de Janeiro, } \\ \text { Brasil }\end{array}\end{array}$

Análise das complicações que algumas pacientes apresentaram após o uso do misoprostol para fim abortivo, e qual o tratamento que receberam após o uso desse medicamento.
O misoprostol foi acessível através de setores formais e informais, mas as dosagens variaram, não correspondiam às diretrizes da

Organização

Mundial da Saúde e eram aparentemente ineficazes, resultando em aborto fracassado, sangramento inteso/hemorragi a, dor intesa e/ou infecção.

Estudo sobre a prática do aborto realizado entre 1603 pacientes internadas em sete hospitais públicos no Rio de Janeiro com diagnóstico relacionado ao aborto, bem como de dados de outros estudos hospitalares.

Cerca de $70 \%$ das mulheres hospitalizadas relataram uso do misoprostol. A maioria das mulheres disse que o uso do medicamento era um experiência negativa por causa da dor ou porque foram necessários (continuação)

\section{Recomendações/} Conclusões incompleto,

Os profissionais da saúde que prestam assistência ao aborto e ao tratamento de complicações do aborto precisam de um treinamento sobre o uso correto do misoprostol e sobre o tratamento das complicações. cuidados

\author{
A experiência de \\ brasileiras com \\ misoprostol é um \\ exemplo de como as \\ mulheres diante de \\ uma gravidez \\ indesejada recorrem \\ ao aborto ilegal, \\ quaisquer que sejam \\ os custos para sua \\ saúde.
}


hospitalares.

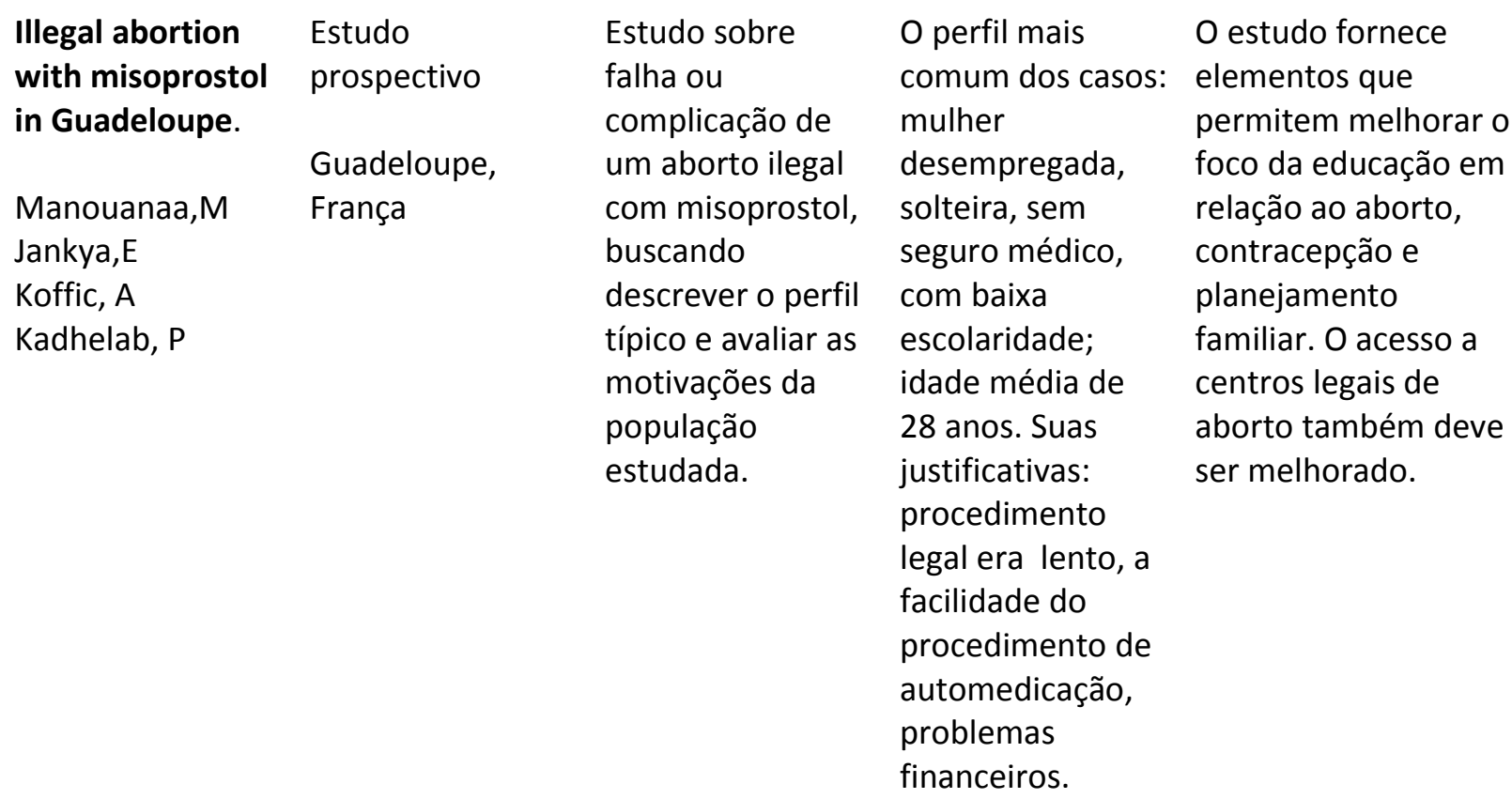

Tabela 1. Apresentação da síntese de artigos incluídos na revisão integrativa.

\begin{tabular}{|c|c|c|c|c|}
\hline & & & & o) \\
\hline $\begin{array}{l}\text { Nome do } \\
\text { artigo/Autores }\end{array}$ & $\begin{array}{l}\text { Tipo/Origem do } \\
\text { estudo }\end{array}$ & $\begin{array}{l}\text { Intervenção } \\
\text { estudada }\end{array}$ & Resultados & $\begin{array}{l}\text { Recomendações/ } \\
\text { Conclusões }\end{array}$ \\
\hline $\begin{array}{l}\text { Post-abortion } \\
\text { Complications } \\
\text { After Interruption } \\
\text { of Pregnancy } \\
\text { With Misoprostol }\end{array}$ & $\begin{array}{l}\text { Faculdade de } \\
\text { Ciências Médicas, } \\
\text { Universidade }\end{array}$ & $\begin{array}{l}\text { Verificar se existe } \\
\text { uma associação } \\
\text { entre o uso de } \\
\text { misoprostol e a } \\
\text { incidência de } \\
\text { complicações }\end{array}$ & $\begin{array}{l}\text { A incidência de } \\
\text { infecção foi } \\
\text { menor nas } \\
\text { mulheres que } \\
\text { declararam ter } \\
\text { usado }\end{array}$ & $\begin{array}{l}\text { Os resultados } \\
\text { sugerem que o } \\
\text { misoprostol deve ser } \\
\text { considerado uma } \\
\text { opção viável em } \\
\text { situações que a }\end{array}$ \\
\hline $\begin{array}{l}\text { Faundes, A } \\
\text { Santos, L.C. } \\
\text { Carvalho, M } \\
\text { Gras, C }\end{array}$ & $\begin{array}{l}\text { Estadual de } \\
\text { Campinas, SP, } \\
\text { Brasil. }\end{array}$ & $\begin{array}{l}\text { sépticas pós- } \\
\text { aborto. }\end{array}$ & $\begin{array}{l}\text { misoprostol do } \\
\text { que outros } \\
\text { métodos } \\
\text { abortivos. }\end{array}$ & $\begin{array}{l}\text { indução do aborto é } \\
\text { legal ou clinicamente } \\
\text { indicada, pois leva a } \\
\text { menos complicações } \\
\text { e, } \\
\text { consequentemente, } \\
\text { menor tempo } \\
\text { hospitalar. }\end{array}$ \\
\hline $\begin{array}{l}\text { Misoprostol } \\
\text { exposure during } \\
\text { the first trimester } \\
\text { of pregnancy: is } \\
\text { the malformation } \\
\text { risk varying }\end{array}$ & 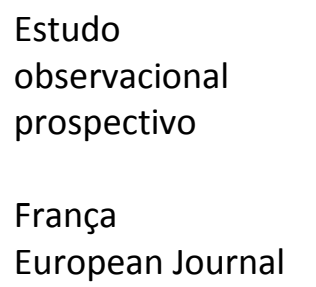 & $\begin{array}{l}\text { Mulheres } \\
\text { expostas ao } \\
\text { misoprostol } \\
\text { durante as } \\
\text { primeiras } 12 \\
\text { semanas de }\end{array}$ & $\begin{array}{l}\text { Foram relatadas } \\
\text { dez malformações } \\
\text { principais }(5,5 \%) \\
\text { (IC95\% 2,65- } \\
\text { 9,82\%) e cinco } \\
\text { delas eram }\end{array}$ & $\begin{array}{l}\text { Os resultados } \\
\text { confirmaram um } \\
\text { padrão específico de } \\
\text { malformações } \\
\text { devido ao uso do } \\
\text { misoprostol no início }\end{array}$ \\
\hline
\end{tabular}




\author{
depending on the of Obstetrics and \\ indication? \\ Auffret, M \\ Gynecology and \\ Reproductive \\ Bernard- \\ Phalippon, N \\ Dekemp, J \\ Carlier, $\mathrm{P}$ \\ Gervoise Boyer, \\ $\mathrm{M}$ \\ Frasco, T \\ Gautier, $S$
}

gravidez e acompanhadas até o parto. consistentes com o padrão de malformações atribuídas ao misoprostol: sequência de Möbius, hidrocefalia, redução transversal terminal dos membros associada a pé torto, sindactilia e encefalocele posterior completo.

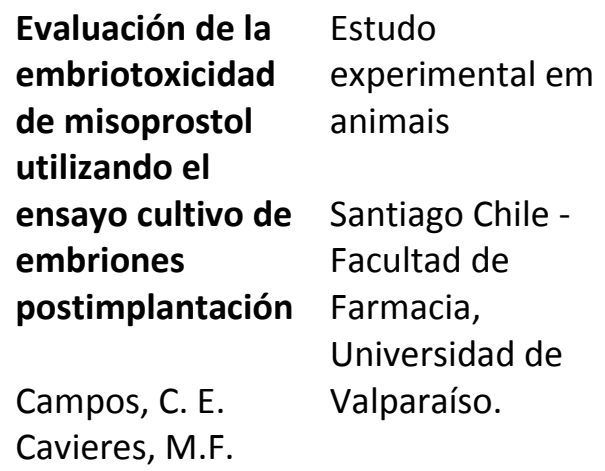

Avaliar a Na maioria dos teratogenicidade do misoprostol usando a cultura de embriões em ratos coletados no início da organogênese e cultivados em soro contendo misoprostol, avaliando parâmetros de funcionalidade, morfologia e morfometria.

\section{embriões, o}

coração

apresentava uma

estrutura irregular

e a cabeça estava pouco

desenvolvida, com evidente atraso na formação do tubo neural, aumento de alterações e atraso no desenvolvimento. da gravidez, mesmo com baixa dosagem da droga. Observouse uma proporção maior de malformações graves em fetos nascidos de mulheres que continuaram a gravidez após falha do aborto voluntário com misoprostol.
Os marcadores de desenvolvimento usados para tabular o escore morfológico total foram marcadamente afetados pelas três concentrações de misoprostol. 
Tabela 1. Apresentação da síntese de artigos incluídos na revisão integrativa.

\begin{tabular}{|c|c|c|c|c|}
\hline & & & & (conclusão) \\
\hline $\begin{array}{l}\text { Nome do } \\
\text { artigo/Autores }\end{array}$ & $\begin{array}{l}\text { Tipo/Origem do } \\
\text { estudo }\end{array}$ & $\begin{array}{l}\text { Intervenção } \\
\text { estudada }\end{array}$ & Resultados & $\begin{array}{l}\text { Recomendações/ } \\
\text { Conclusões }\end{array}$ \\
\hline $\begin{array}{l}\text { Complications of } \\
\text { Unsafe and Self- } \\
\text { Managed } \\
\text { Abortion } \\
\text { Harris, L. H. } \\
\text { Grossman, D. }\end{array}$ & $\begin{array}{l}\text { Revisão } \\
\text { sistemática } \\
\text { Obstetrics and } \\
\text { Gynecology and } \\
\text { the Department } \\
\text { of Women's } \\
\text { Studies, } \\
\text { University of } \\
\text { Michigan, Ann } \\
\text { Arbor (L.H.H.); } \\
\text { and the } \\
\text { Department of } \\
\text { Obstetrics, } \\
\text { Gynecology, and } \\
\text { Reproductive } \\
\text { Sciences, } \\
\text { Advancing New } \\
\text { Standards in } \\
\text { Reproductive } \\
\text { Health (ANSIRH), } \\
\text { and the Bixby } \\
\text { Center for Global } \\
\text { Reproductive } \\
\text { Health, University } \\
\text { of California, San } \\
\text { Francisco. }\end{array}$ & $\begin{array}{l}\text { Informações } \\
\text { sobre os métodos } \\
\text { não } \\
\text { supervisionados } \\
\text { de aborto e suas } \\
\text { implicações. }\end{array}$ & $\begin{array}{l}\text { No mundo todo, } \\
\text { as pessoas } \\
\text { escolhem cada } \\
\text { vez mais } \\
\text { misoprostol para } \\
\text { terminar a } \\
\text { gravidez por } \\
\text { conta própria. } \\
\text { Esse } \\
\text { medicamento é } \\
\text { mais eficaz do } \\
\text { que técnicas } \\
\text { invasivas mais } \\
\text { antigas de aborto } \\
\text { autogestionado. } \\
\text { Por outro lado, } \\
\text { métodos } \\
\text { inseguros podem } \\
\text { precisar de } \\
\text { cuidados } \\
\text { supervisionados. }\end{array}$ & $\begin{array}{l}\text { Tanto a Associação } \\
\text { Americana Médica e } \\
\text { o Colégio Americano } \\
\text { de Obstetras e } \\
\text { Ginecologistas } \\
\text { tomam forte } \\
\text { posições contra a } \\
\text { criminalização do } \\
\text { aborto, porque } \\
\text { impede pacientes } \\
\text { com complicações da } \\
\text { procura de cuidados. }\end{array}$ \\
\hline $\begin{array}{l}\text { Farmacocinética } \\
\text { e } \\
\text { farmacodinâmica } \\
\text { do misoprostol } \\
\text { em Obstetrícia } \\
\text { Souza, A.S.R. } \\
\text { Amorim, } \\
\text { M.M.R.A. } \\
\text { Costa, A.R. } \\
\text { Neto, C.N }\end{array}$ & $\begin{array}{l}\text { Revisão } \\
\text { Sistemática } \\
\text { Centro de } \\
\text { Atenção à Mulher } \\
\text { do Instituto de } \\
\text { Medicina Integral } \\
\text { Prof. Fernando } \\
\text { Figueira (IMIP) - } \\
\text { Recife (PE), Brasil }\end{array}$ & $\begin{array}{l}\text { Revisão } \\
\text { sistemática sobre } \\
\text { o misoprostol e } \\
\text { sua farmacologia. }\end{array}$ & $\begin{array}{l}\text { Observou-se que } \\
\text { o maior pico do } \\
\text { nível plasmático } \\
\text { de misoprostol, } \\
\text { em ordem } \\
\text { decrescente, é do } \\
\text { grupo sublingual, } \\
\text { oral, vaginal com } \\
\text { adição de água e } \\
\text { vaginal, sendo a } \\
\text { biodisponibilidade } \\
\text { também maior na } \\
\text { via sublingual. }\end{array}$ & $\begin{array}{l}\text { Dessa forma, é } \\
\text { imprescindível o } \\
\text { conhecimento da } \\
\text { farmacologia, dos } \\
\text { efeitos e } \\
\text { mecanismos de ação } \\
\text { do misoprostol nas } \\
\text { diferentes formas de } \\
\text { administração, com } \\
\text { base nas evidências } \\
\text { científicas. }\end{array}$ \\
\hline
\end{tabular}

Fonte: Próprio autor, 2020.

Com uma análise detalhada dos artigos da amostra observamos que os principais riscos fetais, a partir do uso inadequado do misoprostol, estão relacionados às malformações, sendo elas:
Síndrome de Mobius, que consiste em paralisia congênita parcial ou completa do VII par craniano, comumente associada à paralisia do nervo abducente e menos frequentemente a 
outros pares de nervos cranianos; hidrocefalia; redução transversal terminal dos membros associada a pé torto; sindactilia e encefalocele posterior completa. Tais anomalias estão presentes mesmo em baixas doses do medicamento. Os casos mais graves estão relacionados aos fetos nascidos de mulheres que continuaram a gravidez após falha no aborto com uso de misoprostol e que não receberam atendimento hospitalar especializado. Entre os artigos selecionados, em um estudo observacional feito com 265 mulheres que realizaram aborto voluntário e foram expostas durante as primeiras 12 semanas de gestação ao misoprostol, relataram-se dez malformações principais (5,5\%) (IC 95\% 2,65-9,82\%), sendo cinco delas compatíveis com o padrão de malformações citado acima e característico do medicamento ${ }^{2}$.

Em relação ao estudo experimental com ratos constatou-se que o misoprostol induziu um efeito embriotóxico dependente da dose, todos os parâmetros avaliados como o diâmetro do saco embrionário, comprimento do crânio, comprimento da cabeça e escore morfológico total diminuíram significativamente com 0 aumento da dose de misoprostol, causando uma diminuição na viabilidade e função embrionárias (desenvolvimento e sobrevida vascular pobre) e alterações morfológicas (principalmente em arcos branquiais, coração e tubo neural). $\mathrm{Na}$ maioria dos embriões estudados, o coração apresentava uma estrutura irregular e a cabeça estava pouco desenvolvida, com evidente atraso na formação do tubo neural do rombencéfalo, mesencéfalo e prosencéfalo, aumento de alterações e atraso no desenvolvimento. Os marcadores de desenvolvimento (fraco desenvolvimento da circulação do saco embrionário, barras braquiais, extremidades anterior e posterior e sistemas óticos e ópticos, entre outros) usados para tabular o escore morfológico total foram marcadamente afetados pelas três concentrações de misoprostol ${ }^{7}$.

O misoprostol possui cinco vias de administração: oral, sublingual, vaginal, bucal (entre a bochecha) e retal. Estudos sugerem que a administração do misoprostol por via vaginal é mais efetiva que a oral na indução do abortamento e na indução do parto, além de apresentar menos efeitos colaterais que as demais vias. Todavia, esses dados foram observados apenas em estudos e ensaios clínicos, e não são citados na bula do Cytotec $^{\circledR}$. No ensaio clínico realizado por Zieman et al. (1997) foram demonstradas as diferenças de absorção do misoprostol nas vias oral e vaginal, constatando que a biodisponibilidade sistêmica do medicamento administrado pela via vaginal foi três vezes maior do que pela via oral ${ }^{1,7}$.

Ao verificar o modo de administração apresentado através da revisão sistematizada sobre misoprostol e sua farmacologia, observouse que o maior pico do nível plasmático, em ordem decrescente, é do grupo sublingual, oral, vaginal com adição de água e vaginal, sendo a biodisponibilidade também maior na via sublingual. Entretanto, o nível é mantido por um maior período de tempo quando a via vaginal é utilizada. Os principais efeitos adversos observados com a administração do misoprostol são calafrios, diarreia, náuseas, vômitos, taquissistolia, hiperestimulação uterina, hipertermia e eliminação de mecônio ${ }^{1}$.

Os resultados apresentados em um estudo qualitativo de Madagascar sugerem fortemente uma falta generalizada de informações entre mulheres e profissionais de saúde sobre o uso correto do misoprostol como abortivo. A idade gestacional em que o medicamento foi usado também variou de algumas semanas a cinco meses, mas quase todas inferiores a doze semanas. $O$ estudo ainda refere sobre complicações que algumas mulheres apresentaram após a tentativa de aborto, relacionando-as à falta de informação tanto da praticante quanto de profissionais da saúde, dando ênfase na importância do conhecimento para ambas as partes. As complicações maternas após o uso incorreto do fármaco abortivo consistiram em hemorragias intensas, dor intensa, perfuração uterina, danos ao trato genital e órgãos internos. Os sinais e sintomas críticos de complicações que demandaram atenção imediata incluíram sangramento vaginal anormal, dor abdominal, infecção e choque circulatório ${ }^{10}$.

\section{DISCUSSÃO}

Quando comparado o uso de misoprostol com outros métodos abortivos, constata-se que o medicamento é mais seguro e eficaz do que as técnicas invasivas mais antigas de aborto autogestionado, levando a menos complicações.

Embora se reconheça a efetividade do misoprostol quando administrado por via oral, sublingual ou retal, as evidências científicas apontam a via vaginal como forma preferencial, 
aplicando-se os comprimidos nos fundos de saco laterais ${ }^{1,7}$. As propriedades farmacocinéticas da via oral e da via vaginal pode explicar por que o misoprostol vaginal é superior. O nível de plasma é mantido em um nível baixo por um longo período de tempo após uma única dose de misoprostol via vaginal, enquanto uma dose semelhante de misoprostol oral resulta em um rápido declínio no nível plasmático após algumas horas. O nível baixo, mas sustentado de misoprostol após a administração vaginal pode exercer um efeito prolongado sobre o útero e resulta em contrações uterinas regulares. Porém, no aborto inseguro, em doses que não correspondem às diretrizes recomendadas pela Organização Mundial de Saúde (OMS) e realizados por profissionais não treinados, o medicamento pode levar a graves consequências.

Em humanos, uma variedade de malformações congênitas foi relatada após uma falha na interrupção da gravidez com exposição ao misoprostol no início da gravidez. O mecanismo pelo qual o medicamento pode causar anomalias é baseado nas contrações uterinas que ele induziu. O misoprostol é um análogo sintético da prostaglandina E1. Na cérvice uterina, as prostaglandinas atuam basicamente sobre a matriz extracelular, com dissolução das fibras colágenas, aumento do ácido hialurônico e aumento do conteúdo de água da cérvice. Além disso, relaxam o músculo liso da cérvice e facilitam a dilatação, ao mesmo tempo em que permitem o acréscimo do cálcio intracelular, promovendo contrações uterinas. Essas contrações causam flexão do embrião, particularmente nos nervos cranianos VI e VII. Pode ocorrer hemorragia e/ou morte celular dos núcleos nestes nervos cranianos, causando a Síndrome de Möebius, a qual é caracterizada por paralisia dos olhos e músculos faciais, microrretrognatia e hipotonia axial ${ }^{8}$.

Desse modo, a Federação Internacional de Ginecologia e Obstetrícia (FIGO), em estudo ecológico envolvendo 46 sociedades membros de diferentes países e continentes, propôs estratégias alternativas correspondentes aos quatro níveis de prevenção: 1) Prevenção de aquisição de fatores etiológicos, significando prevenção de gravidezes indesejadas por meio de informações sobre educação e planejamento familiar; 2) uma vez que a gravidez não intencional / indesejada já ocorreu, prevenção da doença (aborto inseguro), dando acesso a serviços de aborto seguro; 3 ) depois que o aborto inseguro já ocorreu, melhorar a qualidade e o acesso aos cuidados pós-aborto; e 4) impedir a repetição do aborto por meio de aconselhamento em planejamento familiar e contracepção pósaborto ${ }^{11}$.

Visando atender à saúde biopsicossocial das pacientes, torna-se necessário analisar o perfil mais comum das mulheres que se submetem a um aborto inseguro. Um estudo prospectivo europeu que avaliou mulheres em Guadeloupe (Antilhas Francesas) durante um ano apontou para: mulheres desempregadas, solteiras, sem seguro médico, com baixa escolaridade e idade média de 28 anos ${ }^{12}$. No Brasil, segundo dados do Sistema Único de Saúde (SUS), essa população de mulheres são as de cor preta e as indígenas, de baixa escolaridade, com mais de 40 anos ou menos de 14 anos, nas regiões Norte, Nordeste e Centro-Oeste e vivendo sem união conjugal. Acredita-se que esse perfil de faixas vulneráveis é pertinente no mundo todo, entretanto, é imprescindível elaborar estudos com maior tempo de pesquisa, maior número amostral e evidências em diferentes países, de forma a criar políticas públicas adequadas a cada realidade ${ }^{4}$.

O estigma em torno do tema do aborto, e em geral a saúde sexual e o planejamento familiar está inserido nos campos individual, comunitário, religioso e político, e levantam uma barreira que impede o incentivo a pesquisas sobre o tema. Uma revisão sistemática americana, que avaliou as complicações do aborto inseguro, revelou que à medida que as leis se tornam cada vez mais restritivas, as pessoas tendem a interromper a gravidez sem supervisão clínica, impedindo a procura por cuidados em casos de complicações ${ }^{18}$.

As limitações encontradas em alguns estudos relacionam-se ao pequeno número de casos relatados, englobando também falta de critério científico para seleção das pesquisas, informação insuficiente sobre o processo de geração da sequência aleatória para permitir julgamento, levando à susceptibilidade de influência do desfecho. Tais vieses reafirmam a necessidade de maior investimento em pesquisas sobre o tema proposto para que haja maior transparência na avaliação dos riscos relacionados a um aborto inseguro, e consequentemente gerando uma maior atenção para que autoridades políticas de saúde tenham um novo olhar sobre o aborto baseando-se em evidências científicas. 
Com efeito, notamos por meio da revisão integrativa que as mulheres escolhem cada vez mais misoprostol por considerarem a forma mais segura para interromper uma gestação sem apoio médico. De fato, os estudos apontam uma menor taxa de infecção quando comparando o misoprostol com outros métodos abortivos, sendo a administração por via vaginal mais efetiva do que a via oral na indução do abortamento e na indução do parto, além de apresentar menos efeitos colaterais que as demais vias.

No entato, embora seja comprovada cientificamente a segurança do misoprostol em meios hospitalares com respaldo de indicação médica e de profissionais capacitados, os estudos evidenciaram que o uso incorreto e inconsciente do misoprostol pode induzir diretamente alterações no desenvolvimento fetal, levando às malformações e complicações maternas graves, podendo gerar hemorragia severa, sepse e morte.

\section{CONFLITOS DE INTERESSE}

Os autores declaram não haver qualquer potencial conflito de interesse que possa interferir na imparcialidade deste trabalho científico.

\section{REFERÊNCIAS}

1. Souza ASR, et al. Farmacocinética e farmacodinâmica do misoprostol em Obstetrícia. Rev Sist. 2009 Dezembro; 37(12):680-684.

2. Faundes $A$, Comendant $R$, Dilbaz $B$, Jaldesa $G$, Leke R, Mukherjee $B$, et al. Preventing unsafe abortion: achievements and challenges of a global figo initiative. Best Pract. \& Res. Clin. Obst. \& Gyna. [Internet]. 2020 January [cited 2020 Abril 18]; 62:101-112. Available from: http://dx.doi.org/10.1016/j.bpobgyn.2019.05.016

3. Arilha MM. Misoprostol: percursos, mediações e redes sociais para o acesso ao aborto medicamentoso em contextos de ilegalidade no Estado de São Paulo. Ciênc. saúde col. [Internet]. 2012 Julho [citado 2018 Nov. 19]; 17(7):1785-1794. Available from http://www.scielo.br/scielo.php?script=sci arttex t\&pid=S1413-

$\underline{81232012000700017 \& \operatorname{lng}=e n \& n r m=i s o}$

4. Cardoso BB, Vieira FMSB, Saraceni V. Aborto no Brasil: o que dizem os dados oficiais? Cad. Saúde Púb. [Inernet]. 2020 Fevereiro [citado 2020 Abril 18]; 36(1):1-13. Disponivel em: https://doi.org/10.1590/01002$311 \times 00188718$

5. Cecatti JG, Guerra GVQL, Sousa MH, Menezes GMS. Aborto no Brasil: um enfoque demográfico. Rev. Bras. Gineco. Obst. [Internet]. 2010 Março [citado 2020 Abril 25]; 32(3):105$111 . \quad$ Disponível em: http://dx.doi.org/10.1590/s010072032010000300002.

6. Soares, GS. Profissionais de saúde frente ao aborto legal no Brasil: desafios, conflitos e significados. Cad. Saúde Púb. [internet]. 2003 [citado 2020 Abril 26]; 19(2):399-406. Disponível em: $\quad$ http://dx.doi.org/10.1590/s0102$\underline{311 \times 2003000800021 .}$.

7. Zieman $\mathrm{M}$, Fong SK, benowitz MDNL, Banskter D, Darney P. Absorption kinetics of misoprostol with oral or vaginal administration. Obst. and Gyneco. [Internet]. 1997 July [cited 2020 Abril 26]; 90(1):88-92. Available: https://doi.org/10.1016/S0029-7844(97)00111-7

8. Aufrett $\mathrm{M}$, Bernard-Phalippon N, Dekemp J, Carlier P, Boyer MG, Vial T, et al. Misoprostol exposure during the first trimester of pregnancy: Is the malformation risk varying depending on the indication? Euro. Jour. Of Obste. \& Gyneco. And Reprod. Bio. [Internet]. 2016 Dezember [cited 2020 Abril 26]; 207:188-192. Available: https://doi.org/10.1016/i.ejogrb.2016.11.007

9. Carolina Campos E, Cavieres MF. Avaliação da embriotoxicidade do misoprostol utilizando o ensaio de cultura embrionária pósimplantação. Rev. méd. Chile. [Internet]. 2011 Maio [cited 2019 Março 18]; 139(5):613-617. Available:

https://scielo.conicyt.cl/scielo.php?script=sci artt ext\&pid=S0034-

$\underline{98872011000500008 \& \operatorname{lng}=e s \& n r m=i s 0>}$

10. Pourette D, Mattern C, Ratovoson R, Raharimalala P. Complications with use of misoprostol for abortion in Madagascar: between ease of access and lack of information. Contraception. [Internet]. 2018 Februay [cited 2020 Março 15]; 97(2):116-121. Available: http://dx.doi.org/10.1016/j.contraception.2017.1 $\underline{2.005}$ 
11. COSTA SH. Commercial availability of misoprostol and induced abortion in Brazil. Inter. Jour. Of Gyneco. \& Obste. [Internet]. 1998 Dezember [cited 2020 Março 15]; 63(s1):131-139. Available: $\quad$ https://doi.org/10.1016/S00207292(98)00195-7

12. Manouana $M$, Kadhel $P$, Koffi A, Janky $E$. Avortements illégaux par le misoprostol en Guadeloupe. Jour. Gyné. Obsté. Bio. de La Reprod. [Internet]. 2013 abr. [cited 2020 Março 20]; 42(2):137-142. Available from: http://dx.doi.org/10.1016/j.jgyn.2012.10.006.

13. Galvão CM, Sawada NO, Trevizan MA. Revisão sistemática: recurso que proporciona a incorporação das evidências na prática da enfermagem. Rev. Latino-Am Enfer. [Internet]. 2004 maio/junho. [cited 2020 Março 27]; 12(3):549-56. Disponível em: https://doi.org/10.1590/S0104-

$\underline{11692004000300014}$

14. Mendes KDS; Silveira RCCP; Galvão CM. Revisão integrativa: método de pesquisa para a incorporação de evidências na saúde e na enfermagem. Tex. Contex. Enfer. [Internet]. 2008 Dezembro. [citado em 2018 Nov. 19]; 17(4):758$764 . \quad$ Disponivel em: http://www.scielo.br/scielo.php?script=sci arttex $\underline{\mathrm{t} \& \mathrm{pid}=\mathrm{S} 0104-}$

$\underline{07072008000400018 \& \operatorname{lng}=e n \& \mathrm{nrm}=\text { iso }}$

15. Ribeiro OMP, Martins MMFPS, Tronchin DMR. Modelos de prática profissional de enfermagem: revisão integrativa da literatura Rev. Enf. Ref. [Internet]. 2016 Setembro. [citado em 2018 Nov. 20]; IV(10):125-133. Disponível em http://www.scielo.mec.pt/scielo.php?script=sci a rttext\&pid=S0874-

$\underline{02832016000300014 \& \operatorname{lng}=p t \& n r m=i s o}$

16. Kerestes C, Sheets K, Hardy-Fairbanks, CKSJ. Prevalence, attitudes and knowledge of misoprostol for self-induction of abortion in women presenting for abortion at reproductive health clinics. Jour. Sex. Reprod. Heal. Matt. [Internet]. 2019 February. [cited 2020 abr. 27]; 27(1):118-125. Available from: https://doi.org/10.1080/09688080.2019.1571311

17. Faúndes A, Santos LC, Carvalho M, Gras C. Post-abortion complications after interruption of pregnancy with misoprostol. Advan. in Contra. [internet]. 1996 March. [cited 2020 Abril 23]; 12(1):1-9.mar. Available from: https://link.springer.com/article/10.1007/BF0184 9540.

18. Harris LH, Grossman D. Complications of Unsafe and Self-Managed Abortion. New Engl. Jour. Med. [Internet]. 2020 March. [cited 2020 Abril 23]; 382(11):1029-1040. Available from: https://doi.org/10.1056/NEJMra1908412

19. Loureiro DC, Vieira EM. Aborto: conhecimento e opinião de médicos dos serviços de emergência de Ribeirão Preto, São Paulo, Brasil, sobre aspectos éticos e legais. Cad. Saúde Púb. [Internet]. 2004 Maio/junho. [citado em 2020 Maio 5]; 20(3):679-688. Disponível em: https://doi.org/10.1590/S0102$\underline{311 \times 2004000300004}$

20. Fontenelle L, Araujo APQc, Fontana RS. Síndrome de Moebius: relato de caso. Arquivos de Neuro-psiquiatria. [Internet]. 2001 Setembro. [citado em 2020 Março 15]; 59(3):812-814. Disponível em: http://dx.doi.org/10.1590/s0004$\underline{282 \times 2001000500031}$.

21. ALMEIDA MBV, Rolim Filho EL, Malheiros HA, Amaral APCA, Almeida RC, Gusmão AMV. Síndrome de Moebius. Rev. Bras. Orto. [Internet] 2004 Julho. [citado em 2020 Março 15]; 39(7):382-389. Disponível em: https://www.rbo.org.br/detalhes/518/pt-

$\mathrm{BR} /$ sindrome-de-moebius

22. Souza MT, Silva MD. Carvalho R. Revisão integrativa: o que é e como fazer. Einstein [Internet]. 2010 Março. [citado em 2019 Junho 20]; 8(1):102-106. Disponível em: https://www.scielo.br/scielo.php?script=sci artte xt\&pid=S1679$45082010000100102 \& \operatorname{lng}=e n \&$ tlng=en 